\title{
AVALIAÇÃO DE SUCOS DE LARANJA ARTESANAIS PRODUZIDOS NA MICRORREGIÃO DE ERECHIM
}

Assessment of Homemade Orange Juice Produced in Erechim micro region

\author{
Rogério Marcos Dallago ${ }^{1 *}$; Bethina Pascuetti Tres ${ }^{1}$; Andressa Franco Denti ${ }^{1}$; \\ Carolina E. Demaman Oro $^{1}$; Luciana Dornelles Venquiaruto ${ }^{1}$
}

${ }^{1 *}$ Departamento de Engenharia Química e Alimentos, URI - Erechim. E-mail: dallago@uricer.edu.br

Data do recebimento: 06/04/2020 - Data do aceite: 10/06/2020

RESUMO: O suco de laranja tem ganhado cada vez mais espaço no consumo diário da população. Esse fato se deve às suas características sensoriais, propriedades antioxidantes e ao alto teor de vitamina C. Neste sentido, o objetivo do presente estudo foi analisar físico-quimicamente dez amostras de sucos de laranja artesanais, produzidos na microrregião de Erechim/RS. Para tanto, foram realizadas análises de teor de vitamina C (ácido ascórbico), $\mathrm{pH}$, acidez total, sólidos solúveis totais (SST), sólidos totais (ST), índice de maturação, umidade e cor, os quais foram comparados com a legislação vigente. Em relação à caracterização físico-química, todos as amostras analisadas atenderam à legislação em relação aos parâmetros vitamina $\mathrm{C}$, SST (em ${ }^{\circ}$ Brix), acidez em g/100g e cor. A variação de $\mathrm{pH}$ e acidez sugerem que os sucos são produzidos com diferentes variedades de laranjas. Os resultados, principalmente em relação ao teor de vitamina $\mathrm{C}$, confirmam a importância nutricional deste suco, o qual se apresenta como uma alternativa saborosa e saudável para a população.

Palavras-chave: Suco de laranja. Produto artesanal. Análise físico-química.

\begin{abstract}
Orange juice has been gaining more space in the population daily consumption. This fact is due to its sensory characteristics, antioxidant properties and high content of vitamin $\mathrm{C}$. In this sense, the aim of the present work was to physically-chemically analyze ten samples of homemade orange juice, produced in the micro region of Erechim / RS. For this purpose, analysis of vitamin $\mathrm{C}$ (ascorbic acid), $\mathrm{pH}$, total acidity, total soluble solids (TSS), total
\end{abstract}


solids (TS), maturation index, moisture and color content were performed and compared to the current legislation. Regarding the physical-chemical characterization, all analyzed samples complied with the legislation taking into consideration the parameters vitamin C, TSS (in ${ }^{\circ}$ Brix), acidity in $\mathrm{g} / 100 \mathrm{~g}$ and color. The variation in $\mathrm{pH}$ and acidity suggests that the juice is produced with different varieties of oranges. The results confirm the nutritional importance of this juice mainly in relation to the vitamin $\mathrm{C}$ content, which may be considered as a tasty and healthy alternative for the population.

Keywords: Orange juice. Homemade product. Physical-chemical analysis.

\section{Introdução}

A citricultura brasileira apresenta números expressivos que demonstram a grande importância econômica e social que a atividade tem para a economia do país (BARROS, 2016), com destaque para a laranjeira (Citrus sinenses). Especialistas em economia relatam que a citricultura é considerada um dos setores mais competitivos e de maior potencial de crescimento do agronegócio (FAO, 2014). A laranja é um produto de importância para a agricultura e a economia brasileira. À vista disso, o Brasil é responsável por produzir $61 \%$ de todo o suco de laranja consumido no planeta e por exportar $98 \%$ da sua produção, consolidando-se como o mais importante fornecedor global desse produto (NEVES et al., 2017).

A laranja caracteriza-se como uma das frutas de maior consumo em todo o mundo devido ao seu sabor, assim como por seu valor nutricional. As frutas, além de saborosas, são fornecedoras de minerais (potássio, zinco, cobre, magnésio, cálcio, entre outros), vitaminas (especialmente vitamina $C$ ), fibras e outros compostos bioativos, sendo amplamente recomendadas para prevenção de doenças (FAO, 2005; ABOUL-ENEIN et al., 2013). O suco de laranja destaca-se por ser fonte de vitamina $\mathrm{C}$, considerado um poderoso agente antioxidante, mesmo se ingerido em pequenas quantidades (FIGUEREDO et al., 2009; DOLINSKY, 2011).

Vitamina $\mathrm{C}$ é o nome dado ao conjunto de compostos que apresentam atividade biológica semelhante à do ácido L-ascórbico (2,3-enediol-1-ácido glicônico- $\gamma$ lactona) (SPÍNOLA et al., 2013). A importância nutricional dessa vitamina hidrossolúvel está estabelecida há muito tempo. Sabe-se que a mesma participa da síntese de colágeno, atua como antioxidante, facilita a absorção de ferro no trato intestinal e promove a prevenção e cura de resfriados. No entanto, sua deficiência causa escorbuto, enfermidade caracterizada por sangramento da gengiva, dificuldade na cicatrização de feridas, fadiga e anemia, e que pode ser fatal (PHILLIPS et al., 2010).

Segundo Cunha et al. (2014), o ser humano é incapaz de sintetizar ácido ascórbico (vitamina C), dependendo inteiramente da ingestão deste micronutriente. Recomenda-se uma ingestão diária dessa vitamina de $25 \mathrm{mg}$ para crianças, $75 \mathrm{mg}$ para mulheres e de 90 mg para homens (FAO, 2001).

Considerando que as moléculas de ácido ascórbico sofrem facilmente oxidação, é de extrema importância que os sucos produzidos artesanalmente sejam feitos próximos da hora do consumo (já que o mesmo não tem adição de conservantes na sua fabricação) para que os consumidores possam absorver mais eficientemente em seus organismos essa vitamina. 
A produção do suco de laranja in natura produzido artesanalmente é, geralmente, feita manualmente, com emprego de máquinas e utensílios. Nesse processo, o suco de laranja não é submetido a nenhum método de pasteurização e não há nenhum tipo de adição de conservantes, fazendo com que sua vida útil seja reduzida (RUSCHEL et al., 2001; BRITO; ROSSI, 2005). Além disso, a qualidade do suco de laranja é influenciada por fatores microbiológicos, enzimáticos, químicos e físicos, que podem comprometer suas características organolépticas, tais como o aroma, sabor e a cor, além das características nutricionais. Em consequência, esses fatores e as suas possíveis alterações durante o acondicionamento, distribuição e estocagem irão influenciar a vida-de-prateleira do produto. Por isso, as análises físico-químicas são importantes para garantir a qualidade do produto final.

Neste contexto objetivou-se, na presente, pesquisa caracterizar físico-quimicamente amostras de sucos de laranja in natura, produzidos na microrregião de Erechim e comercializados em feiras livres e pequenos comércios alimentícios.

\section{Material e métodos}

Os experimentos foram realizados nas instalações da Universidade Regional e Integrada do Alto Uruguai e das Missões (URI) - Campus Erechim - RS, no laboratório de Bromatologia.

\section{Obtenção das amostras}

Dez amostras de suco de laranja artesanal em embalagens plásticas de $250 \mathrm{~mL}$ foram obtidas em dez estabelecimentos no comércio local de Erechim - RS em janeiro de 2019. Após a coleta, as amostras foram acondicionadas em uma caixa de isopor contendo gelo para evitar perdas devido ao calor ambiente e, então, as mesmas foram devidamente levadas até o laboratório da URI e as análises foram prontamente realizadas. Além das análises analíticas em laboratório, foi examinada a rotulagem apresentada nas embalagens plásticas de $250 \mathrm{~mL}$ comercializadas contendo os sucos de laranja em relação ao Decreto $\mathrm{N}^{\mathrm{o}} 10.026$, de 25 de setembro de 2019, que Regulamenta a Lei $n^{\circ} 13.648$, de 11 de abril de 2018.

\section{Determinação de Vitamina C com iodato de potássio}

A quantificação dos teores de vitamina $\mathrm{C}$ foi realizada segundo o método titulométrico, empregando a técnica de titulação de óxido-redução com o iodato de potássio (iodometria), preconizado pelas normas analíticas do Instituto Adolfo Lutz (2005).

Para cada amostra de suco, foram pipetados $3 \mathrm{~mL}$ de amostra em um erlenmeyer de $250 \mathrm{~mL}$ com $40 \mathrm{~mL}$ de água destilada, seguido da adição de $10 \mathrm{~mL}$ de ácido sulfúrico a $20 \%$. Posteriormente, as amostras foram filtradas e o filtro foi lavado com $10 \mathrm{~mL}$ de água destilada e $10 \mathrm{~mL}$ de ácido sulfúrico a $20 \%$. Após uma etapa de homogeneização adicionou-se $1 \mathrm{~mL}$ de solução de iodeto de potássio a $10 \%$ e $1 \mathrm{~mL}$ de solução de amido $1 \%$ como indicador. Logo após, as amostras foram tituladas com uma solução padrão de iodato de potássio $\left(\mathrm{KIO}_{3}\right) 0,002 \mathrm{~mol} \mathrm{~L}^{-1}$ até observar a formação de uma coloração azul persistente. O teor de ácido ascórbico foi determinado empregando a Equação 1.

Vitamina $C\left(\frac{m g}{100 m L}\right)=\frac{\left(V_{T} \times F\right)}{V_{\alpha}} \times 100$

Sendo: $\mathrm{V}_{\mathrm{T}}=$ Volume de Titulante $\left(\mathrm{KIO}_{3}\right)$ gasto na titulação, em $\mathrm{mL} ; \mathrm{F}=0,886$ (representa a massa de ácido ascórbico, em $\mathrm{mg}$, referente a $1 \mathrm{~mL}$ de $\mathrm{KIO}_{3} 0,002$ mol. $\mathrm{L}^{-1} ; \mathrm{V}_{\mathrm{a}}=$ volume de amostra utilizada, em Ml. 


\section{Sólidos totais e umidade}

Para a análise de sólidos totais, pipetou-se $20 \mathrm{~mL}$ de cada amostra em cápsulas de porcelana previamente preparadas e pesadas com areia do mar. Colocou-se as amostras em banho-maria a $70{ }^{\circ} \mathrm{C}$ até a evaporação da água e concentração da amostra. Após, as cápsulas foram colocadas na estufa a $105^{\circ} \mathrm{C}$ até peso constante.

Depois de transcorrido o tempo necessário em estufa, às cápsulas foram colocadas no dessecador, resfriadas a temperatura ambiente e pesadas novamente. $\mathrm{O}$ experimento foi feito em triplicata para cada amostra.

O cálculo dos sólidos totais foi realizado conforme a Equação 2. O cálculo de umidade foi determinado pela diferença entre a massa inicial e o valor obtido para os sólidos totais.

Sólidos totais $\left(\% \cdot \frac{g}{100 m L}\right)=\frac{(N \times 100)}{V}$

Sendo: $\mathrm{N}=$ (Massa da cápsula com areia do mar vazia, em g) - (Massa da cápsula após dessecação, em g); V = volume de amostra utilizada, em $\mathrm{mL}$.

\section{$\mathrm{pH}$}

$\mathrm{O} \mathrm{pH}$ das amostras foi mensurado utilizando $\mathrm{pHmetro}$ digital (Digimed) previamente calibrado, com imersão do eletrodo diretamente na amostra.

\section{Acidez titulável}

O teor de acidez foi determinado baseado na metodologia descrita pelo Instituto Adolfo Lutz (INSTITUTO ADOLFO LUTZ, 2008), a qual é realizada através da titulação com $\mathrm{NaOH}$ 0,1 mol. $\mathrm{L}^{-1}$ previamente padronizado.

\section{Sólidos Solúveis Totais (SST)}

$\mathrm{O}$ teor de SST foi determinado por refratometria, em ${ }^{\circ}$ Brix a $20^{\circ} \mathrm{C}$. Para a deter- minação de ${ }^{\circ}$ Brix a $20{ }^{\circ} \mathrm{C}$, colocou-se cada suco em uma proveta graduada de $250 \mathrm{~mL}$, onde foi inserido um sacarímetro e realizada a leitura dos ${ }^{\circ}$ Brix. Foi medida a temperatura de cada suco e feita a conversão para ${ }^{\circ}$ Brix a $20^{\circ} \mathrm{C}$.

\section{Índice de maturação}

$\mathrm{O}$ índice de maturação foi calculado como sendo a razão entre os valores médios de ${ }^{\circ}$ Brix a $20^{\circ} \mathrm{C}$ e a acidez para cada amostra.

\section{Análise de cor}

A cor objetiva foi determinada em colorímetro CR-400 Minolta Chromameter (Minolta Cia Ltda.), no espaço CIE L* a* b*, onde $\mathrm{L}^{*}$ é a luminosidade, $\mathrm{a}^{*}$ é a intensidade da cor vermelha e $b^{*}$ é a intensidade da cor amarela (STEWART et al., 1965). O aparelho foi calibrado com uma placa branca, e em seguida as determinações foram realizadas aproximando o aparelho da amostra em uma placa de petri. A leitura foi realizada em triplicata e o valor de Chroma foi calculado conforme a Equação 3.

$$
\text { Chroma }=\sqrt{a^{* 2}+b^{* 2}}
$$

\section{Tratamento estatístico}

Os resultados das análises obtidas foram tratados estatisticamente por análise de variância (ANOVA), seguido de comparação das médias pelo teste de Tukey, com o software Statistica versão 5.0, com nível de confiança de $95 \%$.

\section{Resultados e Discussão}

A Tabela I apresenta os valores obtidos para as determinações de vitamina $\mathrm{C}, \mathrm{pH}$, acidez titulável, Sólidos Solúveis Totais 
(SST) $\left({ }^{\circ}\right.$ Brix a $\left.20{ }^{\circ} \mathrm{C}\right)$ e Índice de maturação (Razão) dos sucos obtidos no comércio local de Erechim-RS.

Com base nos resultados obtidos na Tabela I, pode-se observar que o Estabelecimento 3 , com $88,06 \mathrm{mg} / 100 \mathrm{~mL}$ de suco, foi o que apresentou maior quantidade de ácido ascórbico e diferiu estatisticamente $(p<0,05)$ dos demais estabelecimentos. Os menores valores, com aproximadamente $27 \mathrm{mg} / 100 \mathrm{~mL}$ de suco, são observados para as amostras 2 e 6 . De forma geral, os resultados referentes ao ácido ascórbico encontraram-se na faixa de 27,30 a $45,50 \mathrm{mg} / 100 \mathrm{~mL}$ de suco de laranja, ou seja, todos atendem à legislação, a qual estipula para sucos de laranja um mínimo de $25 \mathrm{mg} / 100 \mathrm{~g}$ de suco (BRASIL, 2018 e 2019) e estão coerentes com a literatura. Silva et al., (2019), encontraram valores de ácido ascórbico (Vitamina C) entre 45,53 e $50,26 \mathrm{mg} / 100 \mathrm{~g}$ para sucos elaborados com diferentes variedades de laranjas.
Sendo a ingestão diária recomendada de vitamina $\mathrm{C}$ de $75 \mathrm{mg}$ para mulheres e 90 mg para homens (IOM, 2000), a vitamina C encontrada em $100 \mathrm{~mL}$ dos sucos analisados para os diferentes estabelecimentos representa, em média, para mulheres e homens adultos, $55 \%$ e $46 \%$ da necessidade diária de ingestão dessa vitamina, respectivamente.

Rosa et al. (2016), analisaram os efeitos do 1-Metilciclopropeno sobre a qualidade pós-colheita de laranjas cv. Pera, armazenadas ao longo de 45 dias à temperatura de $7^{\circ} \mathrm{C}$. Como valor inicial (amostra sem tratamento), os autores encontraram $52,41 \mathrm{mg} / 100 \mathrm{~mL}$ de vitamina $C$, sendo que a amostra controle apresentou variação de 40,25 a 56,76 mg/100 $\mathrm{mL}$ durante o tempo de estudo, valores estes em acordo com os encontrados no presente estudo.

Spínola et al. (2013) avaliaram a concentração de ácido ascórbico em extratos de

Tabela I - Valores de Ácido Ascórbico, pH, Acidez Titulável, Sólidos Solúveis Totais (SST) e Índice de maturação para as amostras de suco de laranja analisadas de cada estabelecimento

\begin{tabular}{cccccc}
\hline $\begin{array}{c}\text { Estabeleci- } \\
\text { mento }\end{array}$ & $\begin{array}{c}\text { Ác. Ascórbico } \\
(\mathbf{m g} / \mathbf{1 0 0} \mathbf{~ m L})^{*}\end{array}$ & $\mathbf{p H}^{*}$ & $\begin{array}{c}\text { Acidez } \\
(\mathbf{g} \text { ác. } \mathbf{C} \text { ítrico } / \mathbf{1 0 0} \\
\mathbf{m L})^{*}\end{array}$ & $\begin{array}{c}\text { SST } \\
\left({ }^{\circ} \text { Brix a 20 }\right. \\
\left.{ }^{\circ} \mathbf{C}\right)^{*}\end{array}$ & $\begin{array}{c}\text { Índice de } \\
\text { maturação }\end{array}$ \\
\hline 1 & $35,22^{\mathrm{bc}} \pm 0,00$ & $3,59^{\mathrm{cd}} \pm 0,18$ & $0,78^{\mathrm{b}} \pm 0,02$ & $10,88^{\mathrm{a}} \pm 0,28$ & 13,94 \\
2 & $27,30^{\mathrm{c}} \pm 1,25$ & $3,78^{\mathrm{c}} \pm 0,13$ & $0,57^{\mathrm{d}} \pm 0,00$ & $10,48^{\mathrm{a}} \pm 0,13$ & 18,38 \\
3 & $88,06^{\mathrm{a}} \pm 0,00$ & $3,60^{\mathrm{cd}} \pm 0,07$ & $0,52^{\mathrm{d}} \pm 0,01$ & $6,88^{\mathrm{cd}} \pm 0,16$ & 13,23 \\
4 & $33,76^{\mathrm{bc}} \pm 6,23$ & $3,54^{\mathrm{cd}} \pm 0,02$ & $0,83^{\mathrm{b}} \pm 0,04$ & $10,56^{\mathrm{a}} \pm 0,20$ & 12,72 \\
5 & $32,29^{\mathrm{bc}} \pm 4,15$ & $4,24^{\mathrm{b}} \pm 0,19$ & $0,37^{\mathrm{e}} \pm 0,02$ & $7,21^{\mathrm{c}} \pm 0,10$ & 19,49 \\
6 & $27,89^{\mathrm{c}} \pm 2,08$ & $6,37^{\mathrm{a}} \pm 0,27$ & $0,05^{\mathrm{f}} \pm 0,01$ & $6,61^{\mathrm{cd}} \pm 0,06$ & 132,20 \\
7 & $42,56^{\mathrm{bc}} \pm 6,23$ & $3,30^{\mathrm{de}} \pm 0,15$ & $0,41^{\mathrm{e}} \pm 0,02$ & $6,01^{\mathrm{d}} \pm 0,19$ & 14,66 \\
8 & $45,50^{\mathrm{b}} \pm 6,23$ & $3,07^{\mathrm{e}} \pm 0,03$ & $1,33^{\mathrm{a}} \pm 0,00$ & $11,36^{\mathrm{a}} \pm 0,31$ & 8,54 \\
9 & $41,09^{\mathrm{bc}} \pm 4,15$ & $3,44^{\mathrm{cde}} \pm 0,04$ & $0,84^{\mathrm{b}} \pm 0,01$ & $8,75^{\mathrm{b}} \pm 0,80$ & 10,42 \\
10 & $42,56^{\mathrm{bc}} \pm 2,08$ & $3,39^{\mathrm{cde}} \pm 0,02$ & $0,71^{\mathrm{c}} \pm 0,01$ & $7,29^{\mathrm{c}} \pm 0,75$ & 10,27 \\
\hline
\end{tabular}

*Médias seguidas de letras iguais não diferem entre si pelo teste de Tukey $(\mathrm{p}<0,05)$, sendo comparadas letras minúsculas entre colunas. 
maracujá, por 5 horas, armazenados em temperatura de $23^{\circ} \mathrm{C}$. Segundo esses autores, não houve queda mensurável da concentração da vitamina após $2 \mathrm{~h}$ de armazenamento, porém perdas de 6,3\% foram detectadas após $5 \mathrm{~h}$ de armazenamento no extrato de maracujá. Para o suco de laranja, Cunha et al. (2014), reportaram que o teor de ácido ascórbico do suco de laranja não apresentou alteração significativa ao longo do período de armazenamento, permanecendo inalterado mesmo após $24 \mathrm{~h}$.

Em relação ao $\mathrm{pH}$, a amostra 6 , com um $\mathrm{pH}$ de 6,37 , foi a que apresentou o maior valor, diferenciando-se acentuadamente das demais amostras, cujos valores de $\mathrm{pH}$ foram entre 3,07 e 4,24. Esta diferença observada entre o $\mathrm{pH}$ da amostra $6 \mathrm{com}$ as demais amostras pode estar relacionada com a(s) variedade(s) da(s) laranja(s) empregada(s) na elaboração dos sucos. Além disso, foi a amostra que apresentou o menor índice de acidez e o maior índice de maturação, o que pode estar relacionado a um suco proveniente de uma laranja mais maturada e com características mais doces. Silva et al. (2019) encontraram valores de $\mathrm{pH}$ de 3,52, 3,30 e 5,83 para sucos de laranja das variedades "Baia", "Common" e "Mimo-do-Céu", respectivamente. Segundo os autores, o elevado $\mathrm{pH}$ observado para a 'Mimo-do-Céu' esta coerente com esta variedade, a qual é caracterizada por apresentar baixa acidez (SOUSA et al., 2016). Neste contexto, os maiores valores de $\mathrm{pH}$ observados para os sucos 5 ( $\mathrm{pH} 4,24)$ e 6 ( $\mathrm{pH} \mathrm{6,37),} \mathrm{os} \mathrm{quais}$ estão coerentes com os menores valores de acidez $(0,37$ e $0,05 \mathrm{~g} / 100 \mathrm{~mL})$ entre os sucos, sugerem que a variedade "Mimo-do-Céu" faz parte de suas composições, nas formas parcial e total, respectivamente.

Para a acidez observam-se valores entre 0,05 (amostra 6) e 1,33 (amostra 8) g de ácido cítrico/100 mL e suco, valores estes que estão coerentes com os seus valores de $\mathrm{pH}(6,37$ e 3,07, respectivamente). Esta tendência era esperada, uma vez que a acidez tende a variar inversamente com o pH do meio. Silva et al. (2019) observaram para diferentes variedades valores de acidez entre 0,08 e $0,98 \mathrm{~g} / 100$ $\mathrm{mL}$, os quais variaram inversamente com os valores de $\mathrm{pH}$ (3,30 e 5,83, respectivamente). Para a maioria dos sucos avaliados os valores de acidez ficaram entre 0,37 e 0,84 g/100 mL, os quais estão coerentes com a literatura. Figueria et al. (2010) encontraram valores médio de acidez entre 0,58 e 0,68 g/100 mL para diferentes sucos de laranja.

O teor de SST, em ${ }^{\circ}$ Brix a $20{ }^{\circ} \mathrm{C}$, variou entre 6,01 e 11,36 . Considerando o limite mínimo de $10,0^{\circ} \mathrm{Brix}\left(\mathrm{a} 20^{\circ} \mathrm{C}\right.$ ), estipulado pela legislação (BRASIL, 2018), somente $40 \%$ das amostras (1, 2, 4 e 8), com valores entre 10,48 e 11,36, atendem a legislação e estão coerentes com a literatura (SILVA et al., 2019; FIGEUIRA et al., 2010). As demais amostras (3, 5, 6, 7, 9 e 10), com valores entre 6,01 e $8,75 \mathrm{~g} / 100 \mathrm{~g}$, não satisfazem a legislação. Contudo, isso pode ser devido ao estágio de maturação das laranjas empregadas na produção dos sucos ou podem ainda sugerir a adição de água no preparo do suco.

Para relação de sólidos solúveis em brix/ acidez, cujo valor mínimo é $7,0 \mathrm{~g} / 100 \mathrm{~g}$ de ácido cítrico anidro, todas as amostras, com valores entre 8,54 e $132,20 \mathrm{~g} / 100 \mathrm{mg}$, satisfazem a legislação vigente (BRASIL, 2018 e 2019). O maior valor, com $132,20 \mathrm{~g} / 100 \mathrm{~g}$, observado para a amostra 6 , está vinculado com sua baixa acidez $(0,05 \mathrm{~g} / 100 \mathrm{~g})$ e reforça a hipótese desta amostra de suco ter sido elaborado com a variedade "Mimo-do-Céu", que na nossa região também é conhecida como "Laranja-do-Céu". Corrobora com esta hipótese o valor de 136,58 \pm 7,21 encontrado por Silva et al. (2019) para esta mesma variedade.

O teor de sólidos totais (ST), umidade e parâmetros de cor são apresentados na Tabela 2 . 
Tabela II - Valores de Sólidos Totais (ST), Umidade e Cor para as amostras de suco de laranja artesanais analisadas no presente trabalho

\begin{tabular}{|c|c|c|c|c|c|c|}
\hline \multirow{2}{*}{$\begin{array}{l}\text { Estabeleci- } \\
\text { mento }\end{array}$} & \multirow{2}{*}{$\begin{array}{c}\mathrm{ST} \\
(\mathrm{g} / 100 \mathrm{~mL}) \\
\end{array}$} & \multirow{2}{*}{$\begin{array}{c}\text { Umidade } \\
(\%)\end{array}$} & \multicolumn{3}{|c|}{ Parâmetros de cor } & \multirow[b]{2}{*}{ Chroma } \\
\hline & & & $\mathrm{L}^{*}$ & $a^{*}$ & $\mathrm{~b}^{*}$ & \\
\hline 1 & $9,19^{\mathrm{c}} \pm 0,28$ & $90,82^{\mathrm{d}} \pm 0,28$ & $33,44^{\mathrm{bc}} \pm 0,77$ & $0,60^{\mathrm{c}} \pm 0,27$ & $22,13^{\mathrm{cd}} \pm 1,87$ & $22,14^{\text {cd }} \pm 1,86$ \\
\hline 2 & $10,67^{\mathrm{a}} \pm 0,19$ & $89,33^{\mathrm{f}} \pm 0,19$ & $32,21^{\mathrm{de}} \pm 0,18$ & $1,20^{\mathrm{b}} \pm 0,04$ & $25,74^{b} \pm 0,38$ & $25,77^{\mathrm{b}} \pm 0,38$ \\
\hline 3 & $7,10^{\mathrm{d}} \pm 0,07$ & $92,90^{\mathrm{c}} \pm 0,07$ & $31,41^{\mathrm{e}} \pm 0,25$ & $1,85^{\mathrm{a}} \pm 0,15$ & $22,51^{\text {cd }} \pm 0,14$ & $22,57^{\mathrm{cd}} \pm 0,15$ \\
\hline 4 & $10,20^{\mathrm{ab}} \pm 0,18$ & $89,80^{\text {ef }} \pm 0,18$ & $32,75^{\mathrm{cd}} \pm 0,25$ & $-1,20^{\mathrm{e}} \pm 0,04$ & $21,50^{\mathrm{d}} \pm 0,41$ & $21,53^{\mathrm{d}} \pm 0,41$ \\
\hline 5 & $6,14^{\mathrm{e}} \pm 0,09$ & $93,86^{b} \pm 0,09$ & $32,90^{\text {cd }} \pm 0,02$ & $-1,02^{\mathrm{e}} \pm 0,01$ & $21,55^{\mathrm{d}} \pm 0,05$ & $21,57^{\mathrm{d}} \pm 0,04$ \\
\hline 6 & $5,60^{\mathrm{ef}} \pm 0,07$ & $94,40^{\mathrm{ab}} \pm 0,07$ & $36,75^{\mathrm{a}} \pm 0,02$ & $-0,07^{d} \pm 0,04$ & $29,22^{\mathrm{a}} \pm 0,03$ & $29,22^{\mathrm{a}} \pm 0,03$ \\
\hline 7 & $5,04^{\mathrm{f}} \pm 0,05$ & $94,96^{a} \pm 0,05$ & $36,73^{a} \pm 0,05$ & $-1,98^{\mathrm{f}} \pm 0,08$ & $21,52^{\mathrm{d}} \pm 0,20$ & $21,61^{\mathrm{d}} \pm 0,19$ \\
\hline 8 & $9,95^{b} \pm 0,15$ & $90,05^{\mathrm{e}} \pm 0,15$ & $36,06^{\mathrm{a}} \pm 0,18$ & $-2,60^{\mathrm{g}} \pm 0,02$ & $22,86^{\mathrm{cd}} \pm 0,14$ & $23,01^{\mathrm{cd}} \pm 0,14$ \\
\hline 9 & $7,38^{d} \pm 0,23$ & $92,62^{c} \pm 0,23$ & $36,21^{a} \pm 0,03$ & $-2,11^{\mathrm{f}} \pm 0,04$ & $27,99^{\mathrm{a}} \pm 0,05$ & $28,07^{\mathrm{a}} \pm 0,05$ \\
\hline 10 & $5,94^{\mathrm{e}} \pm 0,24$ & $94,06^{\mathrm{b}} \pm 0,24$ & $33,96^{\mathrm{b}} \pm 0,01$ & $0,33^{\mathrm{c}} \pm 0,01$ & $23,50^{\mathrm{c}} \pm 0,07$ & $23,49^{\mathrm{c}} \pm 0,06$ \\
\hline
\end{tabular}

Médias seguidas de letras iguais não diferem entre si pelo teste de Tukey ( $\mathrm{p}<0,05)$, sendo comparadas letras minúsculas entre colunas.

Ao analisarmos os sólidos totais e umidade, observamos dois grupos distintos, os quais são coerentes com os SST. As amostras $1,2,4$ e 8 , as quais atendem a legislação vigente (BRASIL, 2018) em relação ao teor de SST, foram as que apresentaram os maiores valores de sólidos totais $(\geq 9,19 \mathrm{~g} / 100$ $\mathrm{mL})$ e menor teor de umidade $(\leq 90 \%)$. Por outro lado, as amostras que não atendem a legislação em relação aos $\operatorname{SST}(3,5,6,7,9$ e 10), foram as que apresentaram os menores teores de ST $(\leq 7,38 \mathrm{~g} / 100 \mathrm{~mL})$ e maiores valores de umidade $(\geq 92,62 \%)$. Contudo, vale ressaltar que a relação ${ }^{\circ}$ Brix/acidez das amostras analisadas encontra-se dentro dos padrões exigidos.

A variação de cor pelo parâmetro a* indica se a cor tende para o vermelho $(+a)$ ou verde (-a). Das amostras de suco analisadas,
$60 \%(4,5,6,7,8$ e 9$)$, com valores negativos (-), tendem para o verde, e $40 \%$ (amostras 1 , 2,3 e 10) com valores positivos (+) tendem para o vermelho.

Quanto ao parâmetro b*, observou-se a presença da cor amarela para todas as amostras, pois os valores entre 21,50 e 29,22 são todos positivos, o que está coerente com as características da fruta. Além disso, os resultados obtidos pelo Chroma também descrevem a coloração amarela.

Em termos de rotulagem, nenhum dos sucos atende ao Decreto $\mathrm{N}^{\mathrm{o}} 10.026$, de 25 de setembro de 2019, que Regulamenta a Lei $\mathrm{n}^{\circ}$ 13.648, de 11 de abril de 2018, que dispõe sobre a produção de polpa e suco de frutas artesanais, pois faltaram informações em relação ao produto comercializado. 


\section{Conclusão}

Os resultados demonstram que os sucos in natura analisados, provenientes de diferentes estabelecimentos na cidade de Erechim-RS, encontram-se em acordo com a literatura e atendem a legislação vigente em relação aos parâmetros físico-químicos de ácido ascórbi- co (Vitamina C), relação de SST (em Brix), acidez em $\mathrm{g} / 100 \mathrm{~g}$ e cor. Somente $40 \%$ das amostras atendem ao parâmetro SST, sugerindo a incorporação de água nas demais amostras. Hipótese esta reforçada pelos baixos valores de sólidos totais $(\leq 7,38 \mathrm{~g} / 100 \mathrm{~mL})$ e elevados valores de umidade $(\geq 92,62 \%)$ observados nas 6 amostras que encontram-se fora dos padrões para SST.

\section{AGRADECIMENTOS}

FAPERGS, CNPQ, CAPES (código de financiamento 001) e URI - Erechim pela infraestrutura e apoio financeiro.

\section{REFERÊNCIAS}

ABOUL-ENEIN, H. Y.; BERCZYŃSK, P.; KRUK, I. Phenolic compounds: the role of redox regulation in neurodegenerative disease and cancer. Mini-Reviews in Medicinal Chemistry, v. 13, n. 3, p. 385-398, 2013.

BARROS, J. R. M.; BARROS, A. L. M.; CYPRIANO, M. P. O mercado da citricultura no Brasil e as suas novas perspectivas. Livro Concecitrus. CitrusBR. 2016

BRASIL. Ministério da Agricultura, Pecuária e Abastecimento. Decreto $\mathbf{N}^{\mathbf{0}} \mathbf{1 0 . 0 2 6}$, de 25 de setembro de 2019, que regulamenta a Lei ${ }^{0} 13.648$, de 11 de abril de 2018, que dispõe sobre a produção de polpa e suco de frutas artesanais em estabelecimento familiar rural. [acesso: abril de 2020]. http://www.in.gov.br/en/web/dou/-/decreto-n-10.026-de-25-de-setembro-de-2019-218271444

BRASIL. Ministério da Agricultura, Pecuária e Abastecimento. Instrução Normativa $n^{\circ} 37$, de $1^{\circ}$ de outubro de 2018. Regulamento técnico geral para fixação dos padrões de identidade e qualidade para suco de fruta. [acesso: abril de 2020]. https://www.gov.br/agricultura/pt-br/assuntos/inspecao/ produtos-vegetal/legislacao-1/biblioteca-de-normas-vinhos-e-bebidas/instrucao-normativa-no-37-de1o-de-outubro-de-2018.pdf/view

BRITO, CRISTIANE SILVEIRA, ROSSI, D.A. Bolores e leveduras, coliformes totais e fecais em sucos de laranja in natura e industrializados não pasteurizados comercializados na cidade de Uberlândia - MG. Bioscience Journal, v. 21, n.1, p. 133-140, 2005. http://www.seer.ufu.br/index. $\mathrm{php} /$ biosciencejournal/article/view/6572/4306

CUNHA, K.D., SILVA, P.R., COSTA, A.L.S., TEODORO, A.J. Estabilidade de ácido ascórbico em sucps de frutas frescos sob diferentes formas de armazenamento. Brazilian Journal Food Technology, v. 17, p. 139-145, 2014.

DOLINSKY, M. Recomendações Nutricionais e Prevenção e Doenças. Rio de Janeiro: Roca, 2011. 160p.

FAO - Food and Agriculture Organization (FAO) Statistics Division, 2014. 
FIGUEIRA, R.; NOGUEIRA, A. M. P.; VENTURINI FILHO, W. G.; DUCATTI, C.; QUEIROZ, E. C.; PEREIRA, A, G, S. Análise Físico-Química e Legalidade em Bebidas de Laranja. Alimentos e Nutrição, v. 21, n. 2, p. 267-272, 2010.

FIGUEREDO, P. P., MARIA, C., SILVA, F., LINHARES, C., PASSOS, X.S., JOSÉ, M., ANTUNES, C. Condições higiênico-sanitárias no preparo de suco de laranja em lanchonetes comerciais de Goiânia , GO. Revista do Instituto de Ciências da Saúde, v. 27, n. 4, p. 374- 377, 2009.

FOOD AND AGRICULTURE ORGANIZATION - FAO. Workshop de Lisboa sobre a promoção de hortofrutícolas nos países de expressão portuguesa: relatório de um workshop conjunto. Geneva: FAO, 2005.

INSTITUTE OF MEDICINE - Panel on Dietary Antioxidants and Related Compounds: Dietary reference intakes: for vitamin $\mathrm{C}$, vitamin $\mathrm{E}$, selenium and carotenoids. Washington: National Academy Press, p. 95-185, 2000.

INSTITUTO ADOLFO LUTZ - IAL. Métodos físico-químicos para análise de alimentos. 4. ed. Brasília: IAL, 2005. 1018 p.

NEVES, M. F.; TROMBIN, V. G. Anuário da citricultura. CitrusBR. São Paulo, 2017.

PHILLIPS, K.M., TARRAGÓ-TRANI, M.T., GEBHARDT, S.E., EXLER, J., PATTERSON, K.Y., HAYTOWITZ, D.B., PEHRSSON, P.R., HOLDEN, J.M. Stability of vitamin C in frozen raw fruit and vegetable homogenates. Journal Food Composition Analytical, v. 23, p. 253-259, 2010.

ROSA, C.I.L.F., CLEMENTE, E., OLIVEIRA, D.M., TODISCO, K.M., DA COSTA, J.M.C. Effects of 1-MCP onthe post-harvest quality of the orange $\mathrm{cv}$. Pera stored under refrigeration. Revista Ciência Agronômica, v. 47, p. 624-632, 2016.

RUSCHEL, C.K., CARVALHO, H.H., SOUZA, R.B. DE, TONDO, E.C. Qualidade microbiológica e físico-química de sucos de laranja comercializados nas vias públicas de Porto Alegre/RS. Food Science and Technology, v. 21, p. 94-97, 2001.

SILVA, A. F.; SILVA, B. M.; SOUSA, A. S. B.; FIGUEIREDO, V. M. A.; MENDONÇA, R. M. N.; SILVA, S. M., Quality, Bioactive Compounds and Antioxidant Activity During Maturation of Oranges Produced in the Borborema Territory. Revista Caatinga, v. 32, n. 2, p. 526-536, 2019. SOUSA, J. R. M.; GHEYI, H. R.; BRITO, M. E. B.; XAVIER, D. A.; FURTADO, G. F., Impact of saline conditions and nitrogen fertilization on citrus production and gas exchanges. Revista Caatinga, v. 29, n. 2, p. 415-424, 2016.

SPÍNOLA, V., MENDES, B., CÂMARA, J.S., CASTILHO, P.C. Effect of time and temperature on vitamin $\mathrm{C}$ stability in horticultural extracts. UHPLC-PDA vs iodometric titration as analytical methods. Food Science and Technology, v. 50, p. 489-495, 2013.

STEWART, M. R.; ZIPSER, M. W.; WATTS, B. M. The use of reflectance spectrophotometry for the assay of raw meat pigments. Journal of Food Science, v. 30, n. 3, p. 464-469, 1965. 
